\title{
Review: patient education and counselling increase preventive behaviours
}

\author{
Mullen PD, Simons-Morton DG, Ramírez G, et al. A meta-analysis of trials evaluating patient education and counseling for three \\ groups of preventive health behaviors. Patient Educ Couns 1997;32:157-73.
}

\section{Questions}

Do patient education and counselling increase preventive behaviours in healthy adults? Which approaches lead to the largest effects?

\section{Data sources}

Studies were identified using Medline, Healthline, Dissertation Abstracts, and PsycINFO; bibliographies of review papers; and contact with experts in the field.

\section{Study selection}

Published and unpublished studies were selected if they measured the effect of counselling or patient education on a preventive health behaviour, studied healthy patients seen in clinical settings in a developed country, used a control group, had $\geqslant 60 \%$ follow up, had $\geqslant 15$ participants in each group at the end of the testing period, reported sufficient data to calculate an effect size, and used intention to treat analysis. Studies were excluded if they tested medication interventions for behaviour change or included patients who had special learning needs or a specific disease.

\section{Data extraction}

Data were extracted on the characteristics of the intervention, the type of behaviour addressed, and the study characteristics.

\section{Main results}

74 studies (67 published from 1972-94; 7 unpublished) met the inclusion criteria. Because of the presence of heterogeneity $(\mathrm{p}<0.001)$, studies were analysed using the random effects model for each of the following 3 behaviour groups: smoking (35 studies) and alcohol misuse (4 studies); nutrition (12 studies) and weight control (5 studies); and other behaviours which included contraceptive use (6 studies), breast self examination (3 studies), exercise (1 study), stressor reduction (2 studies), and injury prevention ( 6 studies). The overall weighted average effect sizes were 0.61 (95\% CI 0.45 to 0.77 ) for alcohol and smoking misuse, 0.51 (CI 0.20 to 0.82 ) for nutrition and weight control, and 0.56 (CI 0.34 to 0.77 ) for other preventive behaviours. Multiple regression analyses showed that for studies on smoking and alcohol misuse, larger effect sizes were associated with nonrandomised designs, a behavioural orientation, the use of media in addition to personal communication, and the use of self monitoring. In studies on nutrition and weight control, larger effect sizes were associated with the use of multiple channels of communication with supportive materials, $\geqslant 30$ day follow up, and the use of self monitoring. In studies on other behaviours, larger effects were associated with low risk status and $<30$ days follow up. Number of contacts and essential principles of education (reinforcement, individualisation, relevance, and feedback) were not associated with larger effect sizes in the regression analyses.

\section{Conclusions}

Patient education and counselling are effective for increasing preventive behaviours in people who are healthy. For smoking and alcohol misuse and for nutrition and weight control, self monitoring leads to more preventive behaviours.

Sources of funding: National Center for Health Services Research and Health Care and Technology Assessment; Henry J Kaiser Family Foundation; US Office of Disease Prevention and Health Promo-

For correspondence: Dr P D Mullen, Center for Health Promotion Research and Development, University of Texas School of Public Health, Houston, TX 77030, USA. Fax +1 7135009602.

A modified version of this abstract appears in ACP Journal Club 1998 May-Jun and Evidence-Based Medicine 1998 May-Jun.

\section{Commentary}

Patient education and counselling may influence the health of populations by potentially preventing disease morbidity and mortality. Although many educational strategies purport to be effective, systematic examination of the outcome of such strategies has been limited.

Mullen et al examined published and unpublished studies of patient education and counselling to determine the effectiveness of primary prevention approaches dealing with specific health behaviours. They identified 74 studies. Although this is a large number of studies for a meta-analysis, the studies included diverse study methods, prevention areas, and intervention approaches, as well as varied lengths of follow up. Many of the individual studies were too small to show consistent effects. By pooling studies across diverse prevention areas, it is possible that the significance of the effect of some interventions in specific prevention areas cannot be discerned.

An important aspect of this study is the way in which data were characterised, condensed, and scored. Based on characteristics associated with behaviour change, interventions were evaluated for their use of principles of education, intervention orientation (cognitive or behavioural), number of patient contacts, channel of communication (personal, media, or both), and self monitoring (present or absent). Overall, the analyses show that patient education and counselling con- tribute to behaviour change for prevention. Nurse practitioners, public health nurses, and others who focus on preventive behavioural change should use behavioural techniques, especially teaching self monitoring, and should include personal communication and written or other audiovisual materials, especially when the goal involves eliminating an existing behaviour (eg, smoking and alcohol use)

Toula M Gerace, RN, MSc
Family Practice Nurse
London Health Sciences Center
Assistant Professor
University of Western Ontario
London, Ontario, Canada

